\title{
Intégrations dans les Amériques: Nouvelles perspectives socio-économiques
}

Regional integration in the Americas. New socio-economic perspectives

Integración de las Américas. Nuevas perspectivas socioeconómicas

\section{Christine Zumello et Jean-Baptiste Velut}

\section{OpenEdition}

\section{Journals}

Édition électronique

URL : https://journals.openedition.org/ideas/103

DOI : 10.4000/ideas.103

ISSN : 1950-5701

Traduction(s) :

New socio-economic perspectives on integration in the Americas - URL : https:// journals.openedition.org/ideas/3031 [en]

Integración en las Américas: nuevas perspectivas socioeconómicas - URL : https:// journals.openedition.org/ideas/3037 [es]

Integrações nas Américas: Novas perspectivas socioeconômicas - URL : https:// journals.openedition.org/ideas/3043 [pt]

\section{Éditeur}

Institut des Amériques

Référence électronique

Christine Zumello et Jean-Baptiste Velut, «Intégrations dans les Amériques: Nouvelles perspectives socio-économiques », IdeAs [En ligne], 1 | 2011, mis en ligne le 26 juillet 2018, consulté le 20 octobre 2022. URL : http://journals.openedition.org/ideas/103 ; DOI : https://doi.org/10.4000/ideas.103

Ce document a été généré automatiquement le 20 octobre 2022

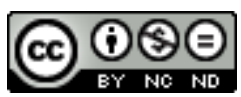

Creative Commons - Attribution - Pas d'Utilisation Commerciale - Pas de Modification 4.0 International - CC BY-NC-ND 4.0

https://creativecommons.org/licenses/by-nc-nd/4.0/ 


\title{
Intégrations dans les Amériques: Nouvelles perspectives socio- économiques
}

\author{
Regional integration in the Americas. New socio-economic perspectives \\ Integración de las Américas. Nuevas perspectivas socioeconómicas
}

Christine Zumello et Jean-Baptiste Velut

1 La dernière décennie du vingtième siècle a été marquée par une accélération des dynamiques d'intégration régionale aux quatre coins du monde. Le plus souvent, le régionalisme s'imposait comme une réponse stratégique à l'intensification de la concurrence internationale dans une économie de plus en plus mondialisée. Le continent américain a vu une myriade de projets d'intégration régionale se décliner sous diverses formes en fonction de leur portée géographique (bilatéralisme/ plurilatéralisme), leur orientation idéologique (néolibéralisme, alternative bolivarienne, etc.), leurs rapports de force (Nord-Sud, Sud-Sud), du degré d'intégration (accord sectoriel, marché commun, zone de libre-échange) et plus généralement, de la "profondeur » des accords régionaux, c'est-à-dire leur caractère contraignant et le pouvoir décisionnel des institutions supranationales.

2 Si ce polymorphisme $\mathrm{du} / \mathrm{des}$ régionalisme(s) dans les Amériques exclut toute conclusion hâtive sur les coûts et les bienfaits de l'intégration régionale, il permet de s'interroger sur les enjeux et les modalités socio-économiques de ces processus. L'intégration économique régionale se confine-t-elle principalement à la fusion des sphères productives et financières? Quelles questions politiques sont considérées comme prioritaires? Quels domaines sont formellement ou officieusement exclus des dynamiques régionalistes?

3 Concernant les modalités du régionalisme, il s'agit d'examiner la structure et le rôle des institutions régionales, qui constituent l'incarnation la plus tangible de la volonté politique accordée à un projet d'intégration. Ainsi, la force d'application d'un accord régional, le fonctionnement et le pouvoir réel des organismes supranationaux, ou le 
budget alloué à un projet sont souvent révélateurs de la vigueur des phénomènes d'intégration. De même, la nature démocratique du processus décisionnel, et en particulier la structure hiérarchique et la dimension participative d'un projet a souvent un impact direct sur sa légitimité, et par conséquent sur sa pérennité.

4 Au delà de ces modalités institutionnelles, les régionalismes se construisent aussi en dehors des sentiers battus de la diplomatie. À l'intégration officielle s'oppose l'intégration « réelle » qui s'appuie sur les flux migratoires, les échanges commerciaux (licites et illicites) et les investissements. Les convergences et divergences entre ces deux formes d'intégration soulèvent, là encore, de nombreuses questions liées à la démocratie, au développement ou à la sécurité. Quelles sont les dynamiques formelles et informelles qui structurent le régionalisme? Peut-on parler de différents modèles d'intégration au sein même du continent américain qui synthétiseraient les relations complexes entre États, marchés, institutions régionales et citoyens, de la même manière que les "modèles de capitalisme $»^{1}$ ont permis de schématiser l'économie politique des États par exemple? De ces modalités découlent les conséquences économiques et sociales des politiques régionalistes, fruits du capital politique investi par les architectes de l'intégration, ou contrecoups inéluctables ou inopinés des processus impulsés.

5 Ce premier numéro de la revue IdeAs se propose d'analyser les enjeux et les modalités socio-économiques de l'intégration régionale dans les Amériques à travers diverses expériences régionales, parmi lesquelles l'ALENA, l'Accord de libre-échange entre les États-Unis et l'Amérique Centrale (ALEAC) ou le Système d'intégration centraméricaine (SICA). Ce dossier rassemble les travaux de chercheurs nord-américains, français et latinoaméricains qui offrent des regards croisés sur les qualités intrinsèques du régionalisme dans les Amériques. L'objectif consiste d'une part à établir un premier bilan socio-économique de ces deux dernières décennies d'intégration au lendemain de la crise financière, dont la propagation au sein du l'hémisphère occidental est directement liée à l'interdépendance croissante des économies du continent. Il s'agit d'autre part de proposer une analyse prospective des principaux défis que devront surmonter les futurs architectes des politiques régionalistes. La mondialisation des chaînes de production rend-elle obsolète le modèle régionaliste ? Quelles solutions les gouvernements du continent américain devront-ils proposer pour combler les lacunes socio-économiques des régionalismes? L'avenir de l'intégration régionale passera-telle par un déclin, un élargissement ou un approfondissement de ces processus ?

6 Ce dossier s'ouvre avec un article de Sherrie Baver, qui se penche sur les enjeux environnementaux de l'intégration régionale en Amérique du Nord et plus précisément sur les liens entre libre-échange et protection environnementale à travers l'expérience de l'ALENA. Sous un angle institutionnaliste, l'auteur analyse l'impact du dispositif institutionnel de l'ALENA sur la mise en place de réformes environnementales au Mexique. L'auteur s'interroge sur le précédent historique créé par les institutions environnementales nord-américaines et leur capacité à réorienter, à long terme, le modèle de libre-échange américain vers une voie plus écologique.

7 Josette Altmann Borbón adopte une approche plus globale des phénomènes d'intégration en Amérique Centrale en se focalisant sur les enjeux et limites du régionalisme, ainsi que sa résilience face à la mondialisation. Elle dresse le bilan du processus de régionalisation au cours des trois dernières décennies et met en exergue les défis sociaux et politiques que les pays centraméricains devront relever pour 
satisfaire les aspirations démocratiques et développementales de leurs peuples. Parmi les paradoxes et les défaillances ou insuffisances du régionalisme centraméricain, elle analyse les conflits entre les différents modèles d'intégration (Nord-Sud, Sud-Sud) au sein de la région, le manque de volonté politique des décideurs ainsi que la fragilité des institutions régionales.

Helen Chang, quant à elle, compare les expériences d'intégration nord-américaine et centraméricaine à travers une analyse économétrique des effets du libre-échange sur la synchronisation des cycles économiques. L'objectif est double. Il s'agit en un premier temps d'évaluer la dépendance des économies latino-américaines vis-à-vis des ÉtatsUnis. En effet, si la synchronisation macroéconomique tend à réduire la volatilité des pays en voie de développement, elle peut aussi accroître leur instabilité en temps de crise par des effets de déversement (spillover effects). En un deuxième temps, l'auteur étudie les solutions à adopter face à ce dilemme en mesurant le degré de coordination des politiques monétaires et budgétaires entre les membres de l'ALENA d'une part, et de l'ALEAC d'autre part.

9 Jean-Baptiste Velut combine également diagnostic et prescription à travers une étude des effets de l'ALENA sur l'économie mexicaine quinze ans après l'entrée en vigueur de l'accord. Dans une analyse de l'évolution des flux de commerce, de l'investissement et des indicateurs sociaux mexicains, l'auteur examine les imperfections du modèle régional nord-américain. Il souligne les paradoxes de l'intégration nord-américaine, et en particulier les conflits entre, d'une part, les modalités institutionnelles et la logique strictement productive de l'ALENA, et d'autre part, les réalités sociales de l'intégration nord-américaine et les limites de l'accord en la matière.

Ces tensions se retrouvent également au cœur du travail de Fanny Lauby, qui analyse les tendances de l'immigration mexicaine vers les États-Unis au cours de la période post-ALENA. Contrairement aux attentes des décideurs nord-américains, l'ALENA n'a pas été en mesure de tempérer les flux de population vers le Nord. Cet échec tient non seulement à l'exclusion de toute clause migratoire au sein de l'accord, mais également à la militarisation de la frontière mexicano-américaine, qui s'est traduite par un phénomène de pérennisation de l'immigration clandestine. L'auteur conceptualise l'émergence d'une forme de régionalisme informel alimenté par les flux d'immigration vers le Nord et les transferts de fonds des migrants vers le Sud. Elle analyse les caractéristiques de ce système parallèle au processus institutionnel de l'ALENA et ses enjeux pour le partenariat États-Unis-Mexique.

11 Ce recueil de travaux issus de traditions universitaires diverses révèle les forces et les faiblesses des processus d'intégration régionale. Il ouvre de nouvelles perspectives et soulève de nombreuses questions quant à l'avenir des régionalismes dans les Amériques, dont les futurs succès passeront incontestablement par une redéfinition des modalités de l'intégration, qui prenne aussi en compte les impératifs sociaux et environnementaux du continent américain. 


\section{NOTES}

1. Peter A. Hall \& David Soskice (eds.), Varieties of Capitalism: the Institutional Foundations of Comparative Advantage, Oxford: OUP, 2001.

\section{AUTEURS}

\section{CHRISTINE ZUMELLO}

Christine Zumello est maître de conférences en civilisation américaine à l'Université de la Sorbonne Nouvelle-Paris 3. Elle travaille plus particulièrement sur les institutions politiques américaines et les questions financières. Elle est membre du CERVEPAS, Centre de Recherche sur la Vie Economique dans les Pays Anglo-Saxons (EA 3960).

\section{JEAN-BAPTISTE VELUT}

Jean-Baptiste Velut est maître de Conférences en Civilisation Américaine à l'Université Sorbonne Nouvelle - Paris III. jean-baptiste.velut@univ-paris3.fr 